(ECM). Hepatic progenitor cell (HPC) activation occurs in the context of severe liver injury. ECM stiffness has been shown to direct differentiation in mesenchymal stem cells. However, the effect of mechanical factors, such as ECM stiffness on HPC responses is poorly characterised. We examined the effect of ECM stiffness on HPC proliferation and differentiation.

Methods Experiments were undertaken using a murine HPC line (BMOL) and primary murine HPCs. Cell culture experiments were performed using a system of laminin-coated polyacrylamide (PA) gel supports of variable stiffness. The stiffness of the PA supports (expressed as shear modulus) was altered across a physiological range $(1-12 \mathrm{kPa})$ corresponding to values encountered in normal and fibrotic livers.

Results Increasing matrix stiffness is associated with enhanced cell spreading. BMOL cells cultured on stiff $(12 \mathrm{kPa})$ supports develop prominent actin stress fibres. The projected surface area (mean\pm SEM) of BMOL cells culture on soft $(1 \mathrm{kPa})$ supports was $378 \pm 21 \mu \mathrm{m}^{2}$ compared to $687 \pm 47 \mu^{2}$ for BMOL cells cultured on stiff $(12 \mathrm{kPa})$ supports $(\mathrm{p}<0.001)$. Cell proliferation (Ki67 positivity) increased as a function of increasing matrix stiffness. The proliferative index (PI) of BMOL cells cultured on $2.5 \mathrm{kPa}$ and $12 \mathrm{kPa}$ supports was 7.1-fold $(p<0.01)$ and 11.8-fold higher $(p<0.001)$, respectively, than cells cultured on $1 \mathrm{kPa}$ supports. Similarly, in experiments with primary cells, the PI of murine HPCs was 1.7-fold higher $(p<0.05)$ when cells were cultured on stiff $(12 \mathrm{kPa})$ vs soft $(1 \mathrm{kPa})$ supports. Quantitative PCR revealed that BMOL cells cultured on soft $(1 \mathrm{kPa})$ supports up-regulate hepatocyte markers, including; albumin (1.5-fold, $\mathrm{p}<0.01)$ and CYP7A1 (1.6-fold, $\mathrm{p}<0.01)$, and down-regulate the HPC/biliary marker cytokeratin-19 (0.6-fold, $\mathrm{p}<0.01)$, relative to cells on stiff $(12 \mathrm{kPa})$ supports. There was no significant change in expression of the biliary epithelial cell markers aquaporin- 1 and $\gamma$-glutamyl-transferase.

Conclusion Physiological changes in ECM stiffness lead to alterations in HPC morphology, proliferation and differentiation. Increased ECM stiffness (as would be encountered in an injured or fibrotic liver) promotes HPC proliferation and expression of the $\mathrm{HPC} /$ biliary marker cytokeratin-19. In contrast, a low-stiffness environment is associated with a reduction in cell proliferation and up-regulation of hepatocyte-specific markers. These results suggest that mechanical factors, such as ECM stiffness might regulate HPC responses following liver injury.

Competing interests None declared.

\section{OC-158 ENDOGENOUS PRODUCTION OF ANTIBIOTICS BY MESENCHYMAL STEM CELLS AND THE POTENTIAL VALUE IN CROHN'S FISTULA HEALING}

doi:10.1136/gutjnl-2012-302514a.158

${ }^{1} \mathrm{~L}$ Meran, ${ }^{*} \mathrm{M}$ Garavaglia, ${ }^{2} \mathrm{~S}$ Heeb, R Bayston, B Richards, ${ }^{3} \mathrm{~W}$ Ashraf, ${ }^{4} \mathrm{~F}$ Rose, ${ }^{4} \mathrm{~K}$ Shakesheff, ${ }^{1} \mathrm{C}$ Hawkey. ${ }^{1}$ Nottingham Digestive Diseases Centre, Nottingham University Hospitals, Nottingham, UK; ${ }^{2}$ Centre for Biomolecular Sciences, Nottingham, UK; ${ }^{3}$ Biomaterials Related Infection Group, University of Nottingham, Nottingham, UK; ${ }^{4}$ Tissue Engineering, STEM group, Centre for Biomolecular Sciences, Nottingham, UK

Introduction Potent effects of human mesenchymal stem cells (MSCs) on immune responses have been demonstrated in vitro studies and animal models, encouraging further investigations into their clinical value. Studies of MSCs in fistula healing have emphasised the immunosuppressive mechanisms of action however, there has been little focus on their antibacterial activity in this setting. We report here on the antibacterial effects of MSCs against Adherent Invasive Escherichia coli (AIEC), reference strain LF82.

Methods Cultured human bone marrow derived MSCs were plated at a density of $5 \times 10^{4}$ cells per square centimetre in 24 well plates and allowed to adhere overnight. Confluent MSCs were preincubated for 1-day with fresh culture medium alone or medium supplemented with $1 \mathrm{ng} / \mathrm{ml}$ TNFa. Cells of passage numbers 4-9 were co-cultured with a calculated 300 Colony Forming Units of AIEC strain LF82 and incubated for $6 \mathrm{~h}$ in a humidified $\mathrm{CO}_{2}$ incubator. Aliquots of the infected medium were taken from each well and plated on LB-agar plates and colonies were counted after overnight incubation. Comparisons were made with aliquots from inoculated wells containing cells that were pre-stimulated with TNF $\alpha$, in addition to wells that did not contain mesenchymal stem cells. Data analyses were performed using the SPSS statistical package (V.19.0).

Results Each of the wells under test conditions was inoculated with $379 \pm 98 \mathrm{CFU}$ at the start of the co-culture time period. In the absence of mesenchymal stem cells, the number of LF82 CFUs in mesenchymal stem cell culture medium had risen to a mean $( \pm S D)$ of $1780 \pm 319$ [n=3], after $6 \mathrm{~h}$. In the presence of passage- 4 MSCs, the number of LF82 CFU was significantly reduced by $81.2 \%$, to $335 \pm 61[n=3] \quad(p<0.05)$. When MSCs were prestimulated with TNF, there was a further reduction of CFU levels to $15 \pm 6[n=3]$. When cells of higher of passage numbers were used, the effects both in the presence and absence of TNF prestimulation diminished such that by passage- 8 , there was no significant inhibition of bacterial growth.

Conclusion The antibacterial properties of MSCs alongside their tissue regenerative potential make them ideal candidates for use in therapeutic strategies for fistula healing. This study demonstrates that in a proinflammatory environment, MSCs enhance bacterial clearance of AIEC. These findings encourage further evaluation of the antimicrobial effector function of MSCs against other strains suspected to participate in the pathogenesis of Crohn's disease.

Competing interests None declared.

\section{BSG information group symposium \& free papers: "Social media and apps: new oppor- tunities, new risks" \\ OC-159 CONTENT ANALYSIS OF ILLNESS BLOGS POSTED ONLINE BY PATIENTS WITH IBD}

doi:10.1136/gutjnl-2012-302514a.159

N Tshuma, ${ }^{*}$ S Aboulela, F Chowdhury, L Macken, N Kamperidis, J Goodhand, J Lindsay. Department of Gastroenterology, Barts and the London NHS Trust, London, UK

Introduction Illness blogs are online accounts of the course of a disease: they are unsolicited first person narratives, that are publicly accessible allowing author-reader interactivity. Expressive writing improves quality of life and scores in patients with irritable bowel syndrome. We sought to compare the accessibility and contents of illness blogs written by patients with ulcerative colitis (UC) and Crohn's disease (CD): hypothesising that psychological distress, reportedly more common in IBD than the general population, and active disease would be the key reasons for a post.

Methods Using the search terms "Crohn's* and Ulcerative colitis* with Blog" and the internet search engine Google, we identified 12 consecutive UC and Crohn's disease illness narratives. All posts written in the preceding year were included. We then undertook a structured quantitative analysis; including an assessment of the readability of posts using the Flesch reading ease (FRE) score and author-reader interactivity. Semi-qualitative analyses of excerpts of the contents of each post were undertaken using Dedoose software to identify narrative themes: wherever possible, for each post, disease activity was recorded.

Results 1297 and 1009 excerpts were made from 642 and 499 posts from the illness blogs written by patients with UC and CD, respectively. There were no differences in the mean [SD] number of 
posts in 1 year (UC: 68.6 [47.5] vs CD: 42.2 [29.6], $p=0.12$ ) or in the number of words per post (UC: 449 [149] vs CD: 475 [263], $p=0.76$ ) between groups. Overall, there was no difference in the mean [SD] readability (FRES) scores (UC: 71 [7.0] vs CD: 67.9 [11.0], $p=0.39$ ): reportedly, easily understood by students aged between 13 and 14 years. No differences were seen in the median number of comments or links per post between groups. Overall, the majority of posts detailed personal experiences of IBD, with no differences between groups (UC: 51\% [326/642] CD: 53\% [264/499], $\mathrm{p}=0.51$ ). Active disease was more frequently coded as a precipitant reason for making an entry in UC 25\% [159/642] vs CD 17\% [84/499], $\mathrm{p}=0.001$. Patients with CD more frequently made entries in order to offer IBD related advice, including recent research advances, than patients with UC (UC: 4\% [26/649] vs CD: 24\% [118/499], $\mathrm{p}<0.0001)$ : whereas patients with UC were more likely to use entries for non-IBD related social networking (UC: $24 \%$ 153/642 vs CD 14\% [72/499], $\mathrm{p}<0.0001)$. In total, only $6 \%(63 / 1141)$ of posts referred to psychological distress, with no differences between groups.

Conclusion The content of blogs differs according to disease type, but relatively few bloggers refer to psychological distress in their posts. Online platforms, such as a blogs, may however be a new way of providing patients with IBD psychological support.

Competing interests None declared.

\section{OC-160 TELEMEDICINE SYSTEMS IN IBD MANAGEMENT-ARE PATIENTS READY?}

doi:10.1136/gutjnl-2012-302514a.160

J Landy, ${ }^{*}$ S T Peake, A Akbar, A L Hart. IBD Unit, St Mark's Hospital, London, UK

Introduction Inflammatory bowel disease (IBD) is a chronic condition characterised by periods of exacerbations and remission, requiring regular medical follow-up. IBD frequently affects patients of working age and the need for life-long follow-up can have significant personal and societal economic implications. Telemedicine systems, could reduce this burden and have been shown to be successful and highly accepted by patients with other chronic diseases. ${ }^{1}$ We aimed to assess the potential technologies by which telemedicine might be employed in our population of IBD patients and the level of patients' acceptance of telemedicine systems in their management.

Methods Patients attending the specialist IBD outpatient clinic were surveyed over a 6-week period. Demographic data, access to technology and acceptance of telemedical systems for the management of their disease were assessed.

Results 52 IBD patients (48\%) responded and completed the survey. $52 \%$ had a diagnosis of UC; $48 \%$ Crohn's disease. $58 \%$ were female. $85 \%$ of patients were aged $18-65$ years and English was the first language in $86 \% .94 \%$ of patients had home access to the internet. $56 \%$ owned smartphones and $52 \%$ used apps regularly. $46 \%$ of patients regularly used web video calling. $85 \%$ of patients wanted electronic access to their personal health data. $65 \%$ and $54 \%$ of patients preferred text or email respectively, to be used for reminders of disease monitoring investigations. $65 \%$ would choose telephone follow-up, while only 38\% would select web based follow-up. $42 \%$ of patients indicated they would undertake web supported self-management of their IBD.

Conclusion IBD patients are of working age and have access to web based and smartphone technologies that could be used in the management of their IBD. IBD patients desire e-health access and the use of technology for communication regarding their disease management as well as web-based monitoring and self management of their IBD. Patients with IBD are ready for telemedicine systems to be employed as an adjunct in the management of their disease.
Competing interests None declared.

\section{REFERENCE}

1. Mclean S, Protti D, Sheikh A. Tele healthcare for longterm conditions. BMJ 2011;342:d120.

\section{Inflammatory bowel disease free papers OC-161 INVESTIGATION OF THE ACTION OF HISTONE DEACETYLASE INHIBITORS IN EX VIVO AND IN VITRO MODELS OF INFLAMMATORY BOWEL DISEASE}

doi:10.1136/gutjnl-2012-302514a.161

${ }^{1} \mathrm{~A} J \mathrm{P}$ Edwards, ${ }^{*}{ }^{1} \mathrm{M}$ Gwiggner, ${ }^{1} \mathrm{~A} \mathrm{G}$ Claridge, ${ }^{1} \mathrm{R} \mathrm{J}$ Morgan-Walsh, ${ }^{2} \mathrm{~A} L$ Hayden, ${ }^{3} \mathrm{~A}$ Bateman, ${ }^{4} \mathrm{~J}$ R F Cummings, ${ }^{2} \mathrm{G}$ K Packham, ${ }^{1} \mathrm{~S}$ L F Pender. ${ }^{1}$ Department of Clinical and Experimental Sciences, Faculty of Medicine, University of Southampton, Southampton, UK; ${ }^{2}$ Department of Cancer Sciences, University of Southampton, Southampton, UK; ${ }^{3}$ Department of Pathology, University Hospital Southampton NHS Foundation Trust, Southampton, UK; ${ }^{4}$ Department of Gastroenterology, University Hospital Southampton NHS Foundation Trust, Southampton, UK

Introduction The management of inflammatory bowel disease (IBD) has evolved rapidly with anti-TNF $\boldsymbol{\alpha}$ agents and the more appropriate use of immunomodulators. Despite this, a large therapeutic gap remains to be filled. Histone deacetylase (HDAC) inhibitors (HDI) preserve acetylation of core histones which prevents chromatin from condensing thus facilitating gene transcription. HDI have been approved for the treatment of sub-cutaneous T-cell lymphoma and recent work has shown that HDI may have a protective antiinflammatory effect in murine models of UC (Tao et al 2007). We hypothesise that HDI have anti-inflammatory effects in human IBD and aim to investigate this using a human ex vivo model of IBD.

Methods Carefully phenotyped patients with active UC $(n=7)$ and $\mathrm{CD}(\mathrm{n}=10)$ undergoing lower GI endoscopy had 8 pinch biopsies $\left(\approx 3 \mathrm{~mm}^{2}\right)$ taken for these experiments. Biopsies were cultured ex vivo at an air liquid interface for $8 \mathrm{~h} \pm$ FK228 or SAHA. Additionally, gut fibroblasts isolated from resection tissue were used to model mucosal plasticity in vitro \pm TNF- $\alpha$ and FK228 and a monocytic cell line (U937) was differentiated in vitro with GMCSF \pm FK228. Supernatants, RNA and tissue were collected for analysis by qRTPCR, mesoscale assay, western blotting and histology.

Results Nanomolar levels of FK228 significantly reduce mRNA expression and protein secretion of Th1 and Th2 cytokines and proinflammatory mediators (IL-8, MMP-1, $-3,-9$ and -12 ) in the ex vivo UC and CD biopsy models. Data suggest that FK228 can decrease mRNA expression of Th1 and Th17 signalling molecules in the ex vivo models. FK228 significantly decreases IL-8, MMP-3 and MMP12 mRNA expression as well as IL-8 and MMP-3 protein secretion by gut fibroblasts and decreases GMCSF induced MMP-12 protein production by U937 cells.

Conclusion This human ex vivo biopsy culture model is relevant for pre-clinical study of drug action in IBD as it utilises small amounts of tissue in a human system. FK228 produces significant antiinflammatory effects at low doses by reducing cytokines and MMPs in this model. FK228 is able to act upon specific cells found in the gut such as lymphocytes, fibroblasts and monocytes to elicit antiinflammatory effects. Based upon our initial findings, further investigation of the role of FK228 in IBD is warranted.

Competing interests None declared.

\section{OC-162 THE ROLE OF MICRORNAS MIR-31 AND MIR-155 IN THE DEREGULATION OF THE IL-13 PATHWAY IN ULCERATIVE COLITIS}

doi:10.1136/gutjnl-2012-302514a.162

${ }^{1} \mathrm{M}$ Gwiggner, ${ }^{*} \mathrm{~A}$ Claridge, ${ }^{2} \mathrm{~F}$ Cummings, ${ }^{1} \mathrm{~T}$ Sanchez-Elsner. ${ }^{1}$ Department of Clinical and Experimental Sciences, University of Southampton, Southampton General Hospital, 